\title{
Optimal Power Allocation for SM-OFDM Systems with Imperfect Channel Estimation
}

\author{
Feng $\mathrm{Yu}^{\mathrm{a}}$, Lijun Song ${ }^{\mathrm{b}}, \mathrm{Xia}_{\mathrm{Lei}^{\mathrm{a}}}$, Yue Xiao ${ }^{\mathrm{a}}$, Zhao Xiang Jiang ${ }^{\mathrm{a}}$, Maozhu Jin ${ }^{\mathrm{c}, *}$ \\ ${ }^{a}$ University of Electronic Science and Technology of China,Chengdu, 610054, P.R. China \\ ${ }^{b}$ Chengdu University of Information Technology, Chengdu 610225, P.R. China \\ ${ }^{c}$ Business School of Sichuan University, Chengdu, 610065, P.R. China
}

\begin{abstract}
This paper analyses the bit error rate (BER) of the spatial modulation orthogonal frequency division multiplex (SM-OFDM) system and derives the optimal power allocation between the data and the pilot symbols by minimizing the upper bound for the BER operating with imperfect channel estimation. Furthermore, we prove the proposed optimal power allocation scheme applies to all generalized linear interpolation techniques with the minimum mean square error (MMSE) channel estimation. Simulation results show that employing the proposed optimal power allocation provides a substantial gain in terms of the average BER performance for the SM-OFDM system compared to its equalpower-allocation counterpart.
\end{abstract}

Keywords: spatial modulation orthogonal frequency division multiplex, channel estimation, power allocation

2010 MSC: 00-01, 99-00

\footnotetext{
${ }^{4}$ Fully documented templates are available in the elsarticle package on CTAN.

*Corresponding author: Maozhu Jin, Business School of Sichuan University, Chengdu, 610065, P.R. China,jinmaozhu@scu.edu.cn

Email addresses: songlj@cuit.edu.cn (Lijun Song), leixia@uestc.edu.cn (Xia Lei), jinmaozhu@scu.edu.cn (Maozhu Jin)

${ }^{1}$ Lijun Song (b. July 3, 1975) is an instructor of college of communication engineering in Chengdu University of Information Technology. His research interests include OFDM and MIMO signal processing.

${ }^{2}$ Maozhu Jin (b. March 14, 1989) received his M.S. degree in the Department of Electronics Science and Technology and Ph.D. in the Department of Business from University of Huazhong Science and Technology, Wuhan, China, in 2005 and 2008, respectively. In April 2009, he joined Business School of Sichuan University, Chengdu, China. His research interests include multi-objective optimization, game theory, service science and supply chain management.
}

Preprint submitted to Journal of LATE $_{E} X$ Templates

November 16, 2015

(C) 2015. This manuscript version is made available under the Elsevier user license http://www.elsevier.com/open-access/userlicense/1.0/ 


\section{Introduction}

Spatial modulation (SM) [1]-[4] is a class of novel efficient and low complexity multiple-input multiple-output (MIMO) technique. In SM-MIMO system, only single transmit antenna is activated to carry the modulated symbol, and the index of the unique active antenna is utilized to carry extra information. The implement and the detection at the transmit and receive sides, especially in massive MIMO environments, are simplified by the special structure of SMMIMO, respectively.

For overcoming the frequency selective channel, SM can be also combined with orthogonal frequency division multiplexing (OFDM) technique [5][6]. SMOFDM owns the advantage of SM-MIMO which alleviates the problem of interantenna synchronization (IAS). In addition, providing a general transmit mode for arbitrary number of transmit and receive antennas, especially for downlink unbalanced massive MIMO-OFDM channels in which the number of transmit antennas is much higher than that of receive antennas, is another advantage of SM-OFDM. Lastly, compared to traditional MIMO techniques such as space time block codes (STBC) and vertical Bell Labs layered space-time (V-BLAST) codes SM-OFDM can offers different tradeoff between spectrum efficiency and transmission performance in long-term evolution (LTE) systems [6]. In general, SM-OFDM is a promising candidate technique in future wireless communications.

In practical scenarios, the channel state information at the receiver (CSIR) is imperfect for imperfections in the channel estimation process. Some recent studies on the performance of MIMO-OFDM systems operating with imperfect CSIR can be found in [7][8]. In [7], Taeyoon Kim et al derive the optimal pilot-to-data power ratio (PDPR) in MIMO-OFDM systems with three different types of pilot patterns, by maximizing the capacity lower bound which is not an appropriate solution in practical wireless communication systems. Then, with least-square (LS) channel estimation and the zero-forcing (ZF) receiver, 
the optimal PDPR was derived in [8] aiming to minimize the average symbol error rate (SER) of MIMO-OFDM system. In addition, the effect of channel imperfections on the performance of SM-OFDM system is presented in [9], however no solution was proposed to reduce the performance degradation due to imperfect CSIR.

35

Based on the above observations, in this paper, we analyse the bit error rate (BERof the SM-OFDM system with minimum mean square error (MMSE) channel estimation and the optimal maximum likelihood (ML) receiver. It is worth noting that the conventional interpolation techniques, such as two order linear interpolation, Gauss interpolation and interpolation based on Wiener filter, and

40 so on, which obtain the channel estimation at the data subcarrier by a weighted linear combination of the channel coefficients of the pilot subcarrier channels essentially, are collectively referred to as the generalized linear interpolation technique in this paper. Then we derive the optimal power allocation by minimizing the upper bound for BER operating with imperfect CSIR. Furthermore, 45 we prove this optimal allocation applies to all generalized linear interpolation techniques with MMSE channel estimation. Finally, we show that employing the proposed optimal power allocation provides a substantial gain in terms of the SM-OFDM systems BER performance compared to that of employing equal power allocation.

50 The remainder of this paper is organized as follows. In the next section, the above SM-OFDM is described in more detail. Section III analyzes the MMSE channel estimation.Section IV analyzes the upper bound for the average BER and derives the optimal PDPR in SM-OFDM system. Section V presents the simulation results, and finally, conclusions are drawn in Section VI.

\section{System Model}

In this paper, we consider a SM-OFDM system with $N_{t}$ transmit and $N_{r}$ receive antennas. Assume the OFDM system is with $N$ subcarriers and the coherent bandwidth contains $N_{c}$ subcarriers. Then,in a coherent bandwidth, 
the $N_{p}$ subcarriers out of $N_{c}$ subcarriers carry pilot symbols, while the rest $N_{d}$ subcarriers carry data symbols. At the transmitter side, the binary data stream is converted to $N$ parallel substreams. After serial-to-parallel conversion, in the $k$ th substreams, $\log _{2} M$ bits select a digital symbol from a unit-energy $M$ order quadrature amplitude modulation ( $M$-QAM) signal set represented by $S=\left\{s_{1}, s_{2}, \cdots, s_{M}\right\}$, while $\log _{2} N_{t}$ bits select an activated antenna out of $N_{t}$ transmit antennas for carrying of the selected symbol. Therefore, the number of simultaneously transmitted bits per channel at a data subcarrier is given by

$$
m=\log _{2} M+\left\lceil\log _{2} N_{t}\right\rceil
$$

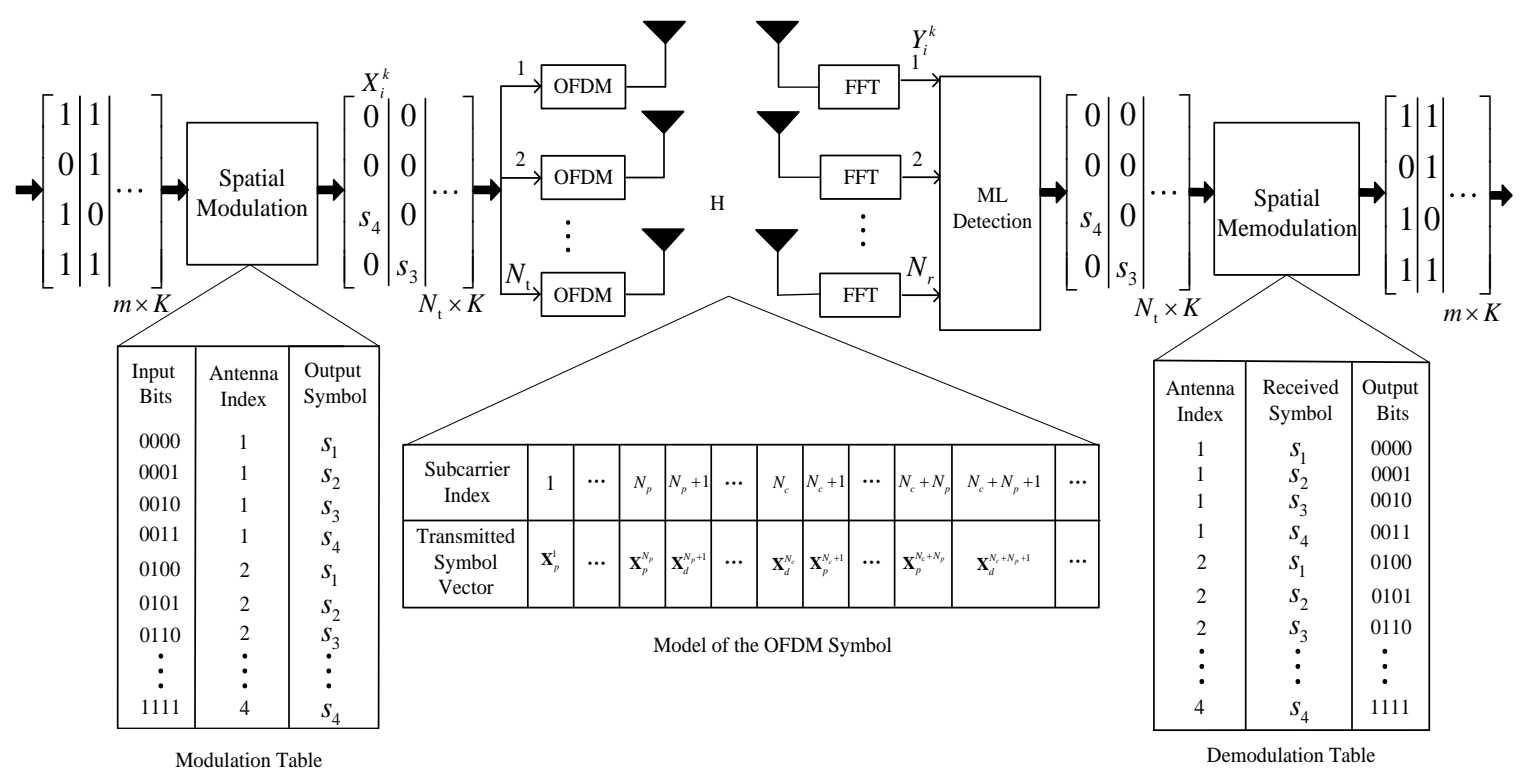

Figure 1: Block diagram of SM- OFDM system.

In the SM-OFDM system, let $\mathbf{X}^{k} \in \mathbb{C}^{N_{t} \times 1}$ represents the transmitted symbol vector, and $\mathbf{H}^{k} \in \mathbb{C}^{N_{r} \times N_{t}}$, given by $\mathrm{H}^{k}=\left[\begin{array}{llll}\mathbf{h}_{1}^{k} & \mathbf{h}_{2}^{k} & \cdots & \mathbf{h}_{N_{t}}^{k}\end{array}\right]$, denote the channel gain matrix with zero-mean, unit variance independent and identical distribution (i.i.d ) complex Gaussian entries, where $\mathbf{h}_{i}^{k}=\left[\begin{array}{llll}h_{1 i}^{k} & h_{2 i}^{k} & \cdots & h_{N_{r} i}^{k}\end{array}\right]^{T}$ 
is the channel gain vector from the $i^{t h}$ transmit antenna to all receive antennas, at the $k^{t h}$ subcarriers. Then the received signal vector $\mathbf{Y}^{k} \in \mathbb{C}^{N_{r} \times 1}$ can be expressed as

$$
\mathbf{Y}^{k}=\mathbf{H}^{k} \mathbf{X}^{k}+\mathbf{Z}^{k}
$$

where $\mathbf{Z}^{k} \in \mathbb{C}^{N_{r} \times 1}$ is the noise vector whose entries are modeled as i.i.d $\mathbb{C} N\left(0, \sigma_{z}^{2}\right)$.

Let $\mathbf{X}_{p}^{k} \in \mathbb{C}^{N_{t} \times 1}$ and $\mathbf{X}_{d}^{k} \in \mathbb{C}^{N_{t} \times 1}$ represent the transmitted pilot symbols vector and data symbols vector respectively at $k$ th subcarrier. Without loss of generality, we employ estimation schemes based on comb-type pilot structure[10]. Transmission model of the system and pilot pattern are shown in Fig.1.

In SM-OFDM system, $N_{p}=N_{t}$, thus, the percentage of the pilot symbols is defined as $\delta=N_{\mathrm{t}} / N_{c}$. The transmission energy for the pilot and data symbols are defined as $E_{p}$ and $E_{d}$, respectively. Therefore, the average transmission energy is

$$
E_{0}=\frac{N_{t} E_{P}+N_{d} E_{d}}{N_{c}}=\delta E_{P}+(1-\delta) E_{d}
$$

Define the energy allocation factor $a$ as [11]

$$
a=\frac{N_{d} E_{d}}{N_{t} E_{P}}=\frac{(1-\delta) E_{d}}{\delta E_{P}} .
$$

Then we obtain

$$
E_{p}=\frac{E_{0}}{\delta(1+a)}
$$

and

$$
E_{d}=\frac{a E_{0}}{(1-\delta)(1+a)} .
$$

$E_{0}$ is assumed to be fixed in the subsequent analysis.

\section{Channel Estimation}

The channel estimation error is studied in this section to see how the energy allocation factor $a$ affects the average BER of the SM-OFDM system operating with an MMSE estimator. 


\subsection{MMSE Channel Estimation at Pilot Subcarrier}

The received symbol matrix for channel estimation can be expressed as follows

$$
\mathbf{Y}_{p}=\mathbf{H}_{p} \mathbf{X}_{p}+\mathbf{Z}_{p}
$$

where $\mathbf{X}_{p}=\left[\begin{array}{llll}\mathbf{x}_{p}^{1} & \mathbf{x}_{p}^{2} & \cdots & \mathbf{x}_{p}^{N_{t}}\end{array}\right]$ is the pilot symbols matrix carried by $N_{t}$ pi-

$70 \quad$ lot subcarriers where $\mathbf{x}_{p}^{i}=\left[0 \cdots x_{p}^{i} \cdots 0\right]^{T}$ and $\left|x_{p}^{i}\right|=1,\left(i=1,2, \cdots, N_{t}\right), \mathbf{H}_{p}=\left[\begin{array}{llll}\mathbf{h}_{1}^{1} & \mathbf{h}_{2}^{2} & \cdots & \mathbf{h}_{N_{t}}^{N_{t}}\end{array}\right]$ is channel matrix at pilot subcarriers, $\mathbf{Z}_{p}=\left[\begin{array}{llll}\mathbf{z}^{1} & \mathbf{z}^{2} & \cdots & \mathbf{z}^{N_{t}}\end{array}\right]$ is noise matrix.

In this paper, we consider the uncorrelated fading scenario, where $E\left\{\mathbf{H}_{p} \mathbf{H}_{p}^{H}\right\}=\mathbf{I}_{N_{t}}$. Thus, the MMSE estimation of the channel $\mathbf{H}_{p}$ is given by[7]

$$
\widehat{\mathbf{H}}_{p}=\frac{\sqrt{E_{p}}}{E_{p}+\sigma_{z}^{2}} \mathbf{Y}_{p} \mathbf{X}_{p}^{H} .
$$

The error correlation matrix can be obtained as

$$
\Psi_{e e}=E\left\{\Delta \mathbf{H}_{p} \Delta \mathbf{H}_{p}^{H}\right\}=\frac{\sigma_{z}^{2}}{E_{p}+\sigma_{z}^{2}} \mathbf{I}_{N_{t}},
$$

where $\Delta \mathbf{H}_{p}=\mathbf{H}_{p}-\widehat{\mathbf{H}}_{p}$. The average estimation mean square error (MSE) is written as

$$
\sigma_{e}^{2}=\frac{1}{N_{t}} \operatorname{trace}\left(\Psi_{e e}\right)=\frac{\sigma_{z}^{2}}{E_{p}+\sigma_{z}^{2}}
$$

Form [11], the entries of channel estimation $\widehat{\mathbf{H}}_{p}$ are modeled as i.i.d $N\left(0,1-\sigma_{e}^{2}\right)$.

\subsection{Channel Estimation at Data Subcarrier}

Consider $I$ order generalized linear interpolation technique, which obtain the channel estimation of $l^{\text {th }}$ data subcarrier by the estimation coefficient of $I$ pilot subcarriers channel which are adjacent to this data subcarrier, and the pilot pattern is shown in Fig.2. Note that conventional interpolation techniques, such as two order linear interpolation, Gauss interpolation and interpolation based on Wiener filter, is linear combination of $\mathbf{H}_{p i}(1 \leq i \leq I)$ essentially. Therefore, channel estimation of the $l^{\text {th }}$ data subcarrier can be written as

$$
\widehat{\mathbf{H}}_{l}=\sum_{i=1}^{I} a_{i}^{l} \widehat{\mathbf{H}}_{p_{i}} .
$$


75 So, the entries of $\widehat{\mathbf{H}}_{l}$ are modeled as i.i.d $\mathbb{C} N\left(0, \sigma_{\hat{h}_{l}}^{2}\right)$, where $\sigma_{\hat{h}_{l}}^{2}=\frac{w_{l} E_{p}}{E_{p}+\sigma_{z}^{2}}$, and $w_{l}=\sum_{i=1}^{I}\left(a_{i}^{l}\right)^{2}$.

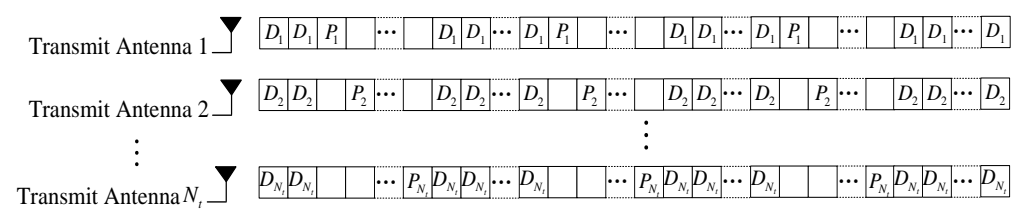

$D_{i}$ :Data symbol transmitted by $i^{\text {th }}$ antenna $\quad P_{i}$ :Pilot symbol transmitted by $i^{\text {th }}$ antenna $\square$ :No symbol

Figure 2: Comb-type pilot structure.

The received symbol vector at the $l^{\text {th }}$ data subcarrier can be expressed as

$$
\mathbf{Y}_{d}^{l}=\sqrt{E_{d}} \widehat{\mathbf{H}}_{l} \mathbf{X}^{l}{ }_{d}+\underbrace{\sqrt{E_{d}} \Delta \mathbf{H}_{l} \mathbf{X}^{l}{ }_{d}+\mathbf{Z}^{l}}_{\mathbf{Z}^{\prime}{ }_{l}},
$$

where $\Delta \mathbf{H}_{l}=\mathbf{H}_{l}-\widehat{\mathbf{H}}_{l}$ is estimation error and with zero-mean, $1-\sigma_{\hat{h}_{l}}^{2}$ variance i.i.d complex Gaussian entries[11], specific $\mathbf{Z}_{l}^{\prime}$ is zero-mean Gussian vector with covariance matrix $\sigma_{\mathbf{z}^{\prime} l}^{2} \mathbf{I}_{N_{r}}$, where

$$
\begin{aligned}
\sigma_{\mathbf{z}^{\prime} l_{l}}^{2} & =\frac{1}{N_{r}} \operatorname{trace}\left\{E\left(\sqrt{E_{d}} \Delta \mathbf{H}_{l} \mathbf{X}_{d}^{l}+\mathbf{Z}^{l}\right)\left(\sqrt{E_{d}} \Delta \mathbf{H}_{l} \mathbf{X}_{d}^{l}+\mathbf{Z}^{l}\right)^{H}\right\} . \\
& =\sigma_{z}^{2}+E_{d}-\frac{w_{l} E_{d} E_{p}}{E_{p}+\sigma_{z}^{2}}
\end{aligned} .
$$

\section{Optimal Power Allocation between Data and Pilot Symbols}

In this section, we analyse the average BER performance of SM-OFDM system with imperfect CSIR and derive the optimal power allocation which 80 minimizes the the average BER of the system.

\subsection{Analysis on Average BER}

Since the channel estimation coefficients of the $l^{\text {th }}$ data subcarrier has the variance $\sigma_{\hat{h}_{l}}^{2}=\frac{w_{l} E_{p}}{E_{p}+\sigma_{z}^{2}}$, the upper bound for the BER of the SM system operating with perfect CSIR in [12] can not be adopted directly. $\widehat{\mathbf{H}}_{l}$ can be rewritten as

$$
\widehat{\mathbf{H}}_{l}=\mathbf{H}_{w} \boldsymbol{\Phi}
$$


where

$$
\mathbf{\Phi}=\sqrt{\frac{w_{l} E_{p}}{E_{p}+\sigma_{z}^{2}}} \mathbf{I}_{N_{t}}
$$

In SM-OFDM system, matrix $E_{p}\left(\mathbf{x}_{i}^{l}-\mathbf{x}_{j}^{l}\right)\left(\mathbf{x}_{i}^{l}-\mathbf{x}_{j}^{l}\right)^{H} \boldsymbol{\Phi}^{H} \mathbf{\Phi}$ rank one due to $\left(\mathbf{x}_{i}^{l}-\mathbf{x}_{j}^{l}\right)\left(\mathbf{x}_{i}^{l}-\mathbf{x}_{j}^{l}\right)^{H}[12]$. Thus, there is only one single non-zero eigenvalue $\varphi_{l}$ , which can be expressed as

$$
\varphi_{l}=\operatorname{trace}\left(E_{d}\left(\mathbf{x}_{i}^{l}-\mathbf{x}_{j}^{l}\right)\left(\mathbf{x}_{i}^{l}-\mathbf{x}_{j}^{l}\right)^{H} \boldsymbol{\Phi}^{H} \mathbf{\Phi}\right)=\frac{w_{l} E_{p} E_{d}}{E_{p}+\sigma_{z}^{2}}\left(\mathbf{x}_{i}^{l}-\mathbf{x}_{j}^{l}\right)^{H}\left(\mathbf{x}_{i}^{l}-\mathbf{x}_{j}^{l}\right) .
$$

Then, the upper bound for the BER of the $l^{\text {th }}$ data subcarrier channel in the SM-OFDM system operating with imperfect CSIR can be expressed as follows $P_{b-M A X}\left(w_{l}\right)=\frac{1}{m 2^{m}} \sum_{i=1}^{2^{m}} \sum_{j=1, j \neq i}^{2^{m}} d\left(\mathbf{x}_{i}^{l}, \mathbf{x}_{j}^{l}\right) \sum_{k=0}^{N_{\mathrm{r}}-1}\left(\begin{array}{c}N_{\mathrm{r}}-1+k \\ k\end{array}\right)\left[1-A_{i j}^{l}\right]^{k}\left(A_{i j}^{l}\right)^{N_{\mathrm{r}}}$,

where $d\left(\mathbf{x}_{i}^{l}, \mathbf{x}_{j}^{l}\right)$ represents the Hamming distance between $\mathbf{x}_{i}^{l}$ and $\mathbf{x}_{j}^{l}$,

$$
\begin{gathered}
A_{i j}^{l}=\gamma\left(\frac{\frac{E_{P} E_{d} w_{l}}{E_{P}+\sigma_{z}^{2}} \lambda_{i j}^{l}}{4 \sigma_{\mathbf{z}^{\prime}}^{2}}\right), \\
\lambda_{i j}^{l}=\left(\mathbf{x}_{i}^{l}-\mathbf{x}_{j}^{l}\right)^{H}\left(\mathbf{x}_{i}^{l}-\mathbf{x}_{j}^{l}\right), \\
\gamma(x)=0.5(1-\sqrt{x /(1+x)}) .
\end{gathered}
$$

Therefore, the upper bound for the average BER of SM-OFDM is given by

$$
P_{b} \leq \bar{P}_{b-M A X}=\frac{1}{N_{d}} \sum_{l=1}^{N_{d}} P_{b-M A X}\left(w_{l}\right) .
$$

\subsection{Optimal Power Allocation}

In (21), the upper bound for the BER is expressed as a function of $E_{p}, E_{d}$, $\sigma_{z}^{2}$ and $w_{l}$. Since the $E_{p}$ and $E_{d}$ are the function of $a$ and $\delta$, the upper bound is in turn expressed as an function in $a, \delta, \sigma_{z}^{2}$ and $w_{l}$. Then, given a fix pilot 
percentage $\delta$, the optimum energy allocation factor, that minimizing the upper bound, can be obtained by solving the equation

$$
\frac{\partial \bar{P}_{b-M A X}}{\partial a}=0 .
$$

Theorem:the optimum power allocation factor is

$$
a^{*}=\sqrt{\frac{\left(\delta(1-\delta)+(1-\delta) r_{0}\right)}{\left(\delta(1-\delta)+\delta r_{0}\right)}}
$$

where $r_{0}=\frac{E_{0}}{\sigma_{z}^{2}}$ represent the average signal-to-noise ratio (SNR).

Proof Define $\left.B_{i j}^{l}=\sqrt{\left(S_{i j}^{l} /\left(1+S_{i j}^{l}\right)\right)}\right)$, where $S_{i j}^{l}=\frac{\frac{E_{P} E_{d} w_{l}}{E_{P}+\sigma_{z}^{2}} \lambda_{i j}^{l}}{4 \sigma_{\mathbf{z}^{\prime}}^{2}{ }_{l}}$. Absolutely, $0<B_{i j}^{l}<1$. According to $E_{p}=\frac{E_{0}}{\delta(1+a)}$ and $E_{\mathrm{d}}=\frac{a E_{0}}{(1-\delta)(1+a)}, B_{i j}^{l}$ can be expressed as

$$
B_{i j}^{l}=\sqrt{\frac{a E_{0}^{2} w_{l} \lambda_{i j}^{l}}{4\left(\delta(1-\delta)(1+a)^{2} \sigma_{z}^{2} \sigma_{z}^{2}+(1-\delta)(1+a) E_{0} \sigma_{z}^{2}+\sigma_{z}^{2} \delta(1+a) a E_{0}+a E_{0}^{2}-a E_{0}^{2} w_{l}\right)+a E_{0}{ }^{2} w_{l} \lambda_{i j}^{l}} .}
$$

Then, The first derivative of $B_{i j}^{l}$ with respect to $a$ can be written as

$$
\frac{\partial B_{i j}^{l}}{\partial a}=2 F_{i j}^{l} G
$$

where

$$
F_{i j}^{l}=\frac{\sqrt{\frac{E_{0}^{2} w_{l} \lambda_{i j}^{l}}{a}}}{\left(4\left(\delta(1-\delta)(1+a)^{2} \sigma_{z}^{2} \sigma_{z}^{2}+(1-\delta)(1+a) E_{0} \sigma_{z}^{2}+\sigma_{z}^{2} \delta(1+a) a E_{0}+a E_{0}{ }^{2}-a E_{0}{ }^{2} w_{l}\right)+a E_{0}{ }^{2} w_{l} \lambda_{i j}^{l}\right)^{1.5}},
$$

$$
G=\left(\delta(1-\delta) \sigma_{z}^{2} \sigma_{z}^{2}+\sigma_{z}^{2} \delta E_{0}\right) a^{2}-\left(\delta(1-\delta) \sigma_{z}^{2} \sigma_{z}^{2}+(1-\delta) E_{0} \sigma_{z}^{2}\right) .
$$

For $\mathrm{A}_{i j}^{l}=0.5\left(1-\mathrm{B}_{i j}^{l}\right), \frac{\partial A_{i j}^{l}}{\partial a}=F_{i j}^{l} G$. Then, the first derivative of the upper bound for the BER of the $l^{\text {th }}$ data subcarrier channel with respect to $a$ can be written as

$$
\frac{\partial P_{b-M A X}\left(w_{l}\right)}{\partial a}=\frac{G}{m 2^{m}} \sum_{i=1}^{2^{m}} \sum_{j=1, j \neq i}^{2^{m}} d\left(\mathbf{x}_{i}, \mathbf{x}_{j}\right) \sum_{k=0}^{N_{\mathrm{r}}-1}\left(\begin{array}{c}
N_{\mathrm{r}}-1+k \\
k
\end{array}\right) F_{i j}^{l} \beta_{l, i j}^{\mathrm{k}}
$$


where

$$
\beta_{l, i j}^{\mathrm{k}}=\left(A_{i j}^{l}\right)^{N_{\mathrm{r}}-1}\left(1-A_{i j}^{l}\right)^{k-1}\left(N_{r}\left(1-A_{i j}^{l}\right)-k A_{i j}^{l}\right)
$$

Since $1-A_{i j}^{l}>0.5>A_{i j}^{l}, N_{r}\left(1-A_{i j}^{l}\right)-k A_{i j}^{l}>0,\left(A_{i j}^{l}\right)^{N_{\mathrm{r}}-1}>0,\left(1-A_{i j}^{l}\right)^{k-1}>0$, $\forall k=0,1,2, \ldots \ldots N_{\mathrm{r}}-1$ and $\beta_{l, i j}^{\mathrm{k}}>0$. In addition, when $i \neq j, \lambda_{i j}=\left(\mathbf{x}_{i}-\mathbf{x}_{j}\right)^{H}\left(\mathbf{x}_{i}-\mathbf{x}_{j}\right)>0$ and $d\left(\mathbf{x}_{i}, \mathbf{x}_{j}\right)>0$. Therefore

$$
\frac{\partial P_{b-M A X}\left(w_{l}\right)}{\partial a}=0 \Leftrightarrow \mathrm{G}=0
$$

Furthermore, the first derivative of the upper bound for the BER of the SMOFDM system with respect to $a$ is expressed as

$$
\frac{\partial \bar{P}_{b-M A X}}{\partial a}=\frac{1}{N_{d}} \sum_{l=1}^{N_{d}} \frac{\partial P_{b-M A X}\left(w_{l}\right)}{\partial a} .
$$

According to the (28), (31) can be rewritten as

$$
\frac{\partial P_{b-M A X}}{\partial a}=\frac{G}{N_{\mathrm{d}}} \sum_{l=1}^{N_{\mathrm{d}}} \frac{1}{m 2^{m}} \sum_{i=1}^{2^{m}} \sum_{j=1, j \neq i}^{2^{m}} d\left(\mathbf{x}_{i}, \mathbf{x}_{j}\right) \sum_{k=0}^{N_{\mathrm{r}}-1}\left(\begin{array}{c}
N_{\mathrm{r}}-1+k \\
k
\end{array}\right) F_{i j}^{l} \beta_{l, i j}^{\mathrm{k}} .
$$

Based on the above analysis,

$$
\frac{\partial P_{b-M A X}}{\partial a}=0 \Leftrightarrow G=0
$$

Given the energy allocation factor $a>0$, the solution of equation $G=0$ should be

$$
a^{*}=\sqrt{\frac{\left(\delta(1-\delta)+(1-\delta) r_{0}\right)}{\left(\delta(1-\delta)+\delta r_{0}\right)}}
$$

When $0<a<a^{*}, \frac{\partial P_{b-M A X}}{\partial a}<0$ and $\frac{\partial P_{b-M A X}\left(w_{l}\right)}{\partial a}<0$ due to $G<0$,In addition, when $a>a^{*}, \frac{\partial P_{b-M A X}}{\partial a}>0$ and $\frac{\partial P_{b-M A X}\left(w_{l}\right)}{\partial a}>0$ due to $G>0$. Thus, $a^{*}$ is the point where $P_{b-M A X}$ and $P_{b-M A X}\left(w_{l}\right)$ are minimized.

Above analysis process illustrates the optimal factor that minimizing the upper bound for the average BER of SM-OFDM system and that minimizing the upper bound for the average BER of a specific subcarrier are both obtained

by solving the equation $G=0$. In addition, when employing a same kind of generalized linear interpolation technique, the estimation error of different data 
subcarrier channel is different due to the coefficient $w_{l}, l=1,2, \cdots, N_{d}$, Furthermore, the coefficient $w_{l}$ at the same data subcarrier is vary with the change of generalized linear interpolation technique. However, the optimal factor $a^{*}$ is not related to the coefficient $w_{l}$.

Consequently, we can come to the following conclusion: whether employing any kind of generalized linear interpolation technique, the optimal factor is still $a^{*}$ which also minimizes the upper bound for the average BER of any specific data subcarrier channel.

\section{Simulation Results}

In this section, we study the BER performance of the SM-OFDM system operating with imperfect CSIR, and the BER improvement owing to the proposed optimum energy allocation over that of equal power allocation between the pilot and data symbols.

\subsection{Simulation Scenario}

In all our simulations, employ LTE standard channel ITU-PedA, and the parameters of channel are listed in the table 1. while the parameters of SM-OFDM system, referencing to the LTE standard, are listed in table 2 . In addition, the imperfect CSIR scenario relies on the MMSE channel estimation. For convenience, the parameters in Fig.4, 5, 6, 7 are summarized as follows, $N_{d}=100$, $N_{t}=N_{r}=4, \delta=\frac{N_{t}}{N_{t}+N_{d}}$ and $r_{0}=15 \mathrm{~dB}$.

Table 1: Parameters of Channel ITU-PedA

\begin{tabular}{|c|c|c|c|c|}
\hline Average Power(dB) & 0 & -9.7 & -19.2 & -22.8 \\
\hline Relative Time Delay(us) & 0 & $1.10 \mathrm{e}-7$ & $1.90 \mathrm{e}-7$ & $4.10 \mathrm{e}-7$ \\
\hline
\end{tabular}

\subsection{Simulation Analysis}

In Fig.3, the analytical BER of SM-OFDM system is plotted given in (21) as a function of the factor $a$ without employing linear interpolation technique. 
Table 2: Parameters of SM-OFDM System

\begin{tabular}{|c|c|c|c|c|}
\hline$N$ & Bandwidth & Subcarrier Intervals & Cyclic Prefix $(\mathrm{CP})$ & $M$ \\
\hline 1200 & $20 \mathrm{MHz}$ & $15 \mathrm{kHz}$ & Conventional Cyclic Prefix & 4 \\
\hline
\end{tabular}

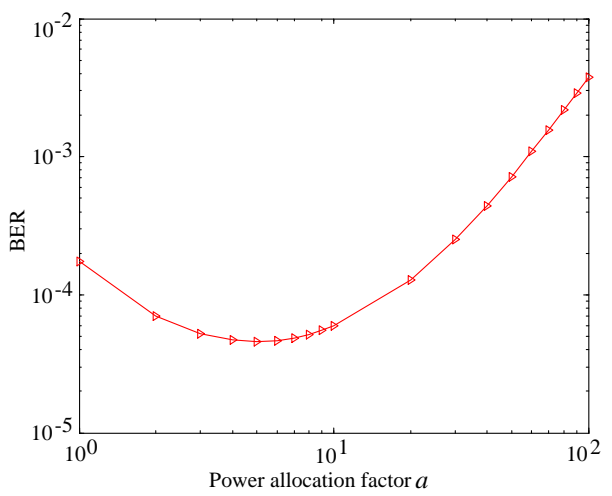

Figure 3: The effect of the factor $a$ on the upper bound for BER of SM-OFDM system, which is computed from (21), without employing linear interpolation technique.

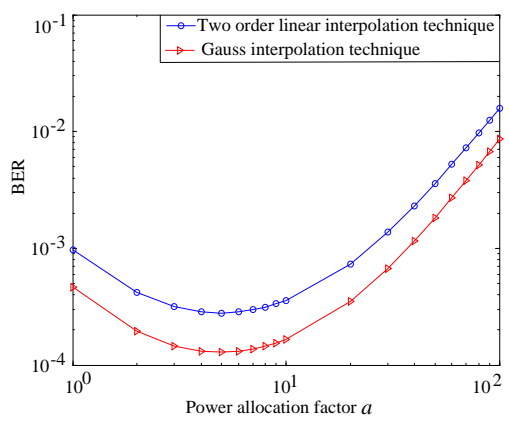

Figure 4: Comparison of the upper bound for BER as a function of the factor $a$ employing Gauss interpolation technique with that of employing two order linear interpolation technique, in SM-OFDM system. 


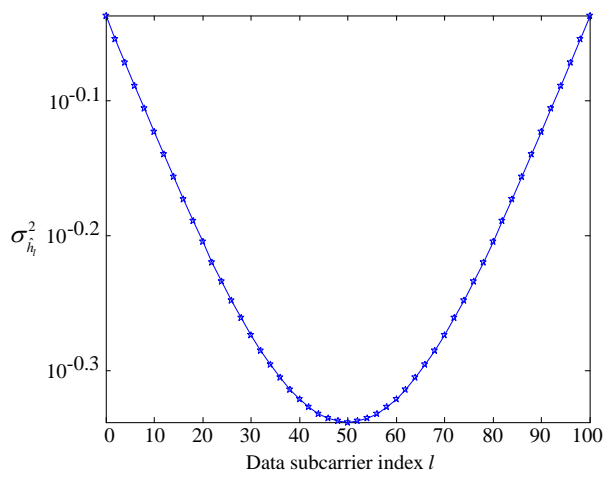

Figure 5: $\sigma_{\hat{h}_{l}}^{2}$ as a function of the subcarrier index $l$.

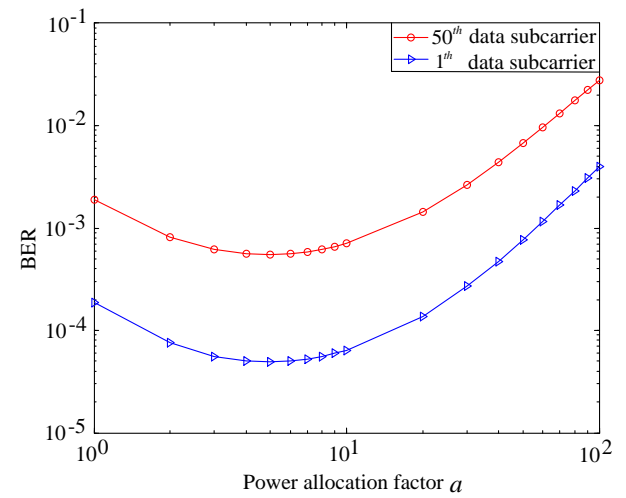

Figure 6: Comparison of the upper bound for BER as a function of the factor $a$ of the best data subcarrier channel with that of the worst data subcarrier channel, in a coherent bandwidth. 


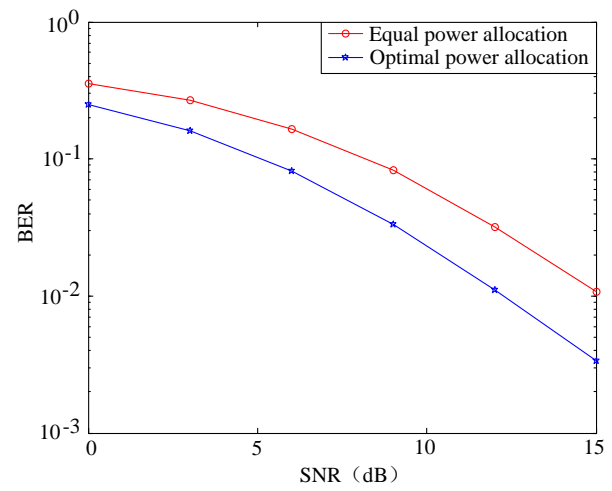

(a)

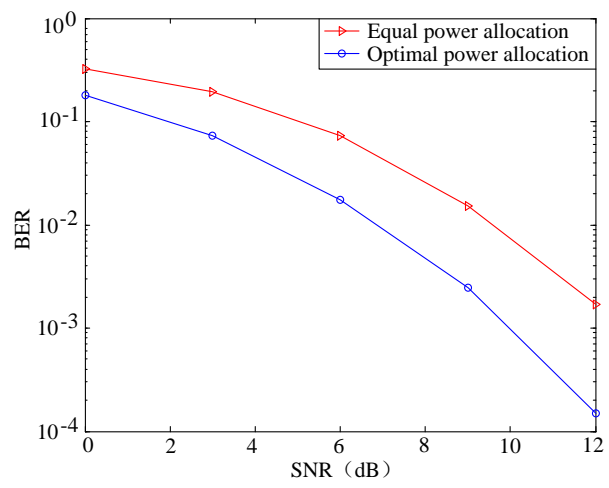

(b)

Figure 7: Compares the simulated BER of the SM-OFDM system operating with imperfect CISR employing the proposed optimal power allocation with that of employing the equal power allocation between data and pilot symbols. Plot (a) corresponds to $N_{t}=N_{r}=2$ case and Plot (b) corresponds to that of $N_{t}=N_{r}=4$. 
Then, in Fig.4, the analytical BER is plotted as a function of $a$ when using Gauss interpolation technique and two order linear interpolation technique respectively. By carefully observing the curves in Fig.3 and Fig.4, we find that the optimal energy allocation factor for a given value of $\delta$ was around $a=5$, where the SM-OFDM system achieves the best BER performance. This observation implies $a^{*}$ is optimal for the SM-OFDM system when employing the generalized linear interpolation technique.

From the results of Fig.5, we observe that the $\sigma_{\hat{h}_{l}}^{2}$ of data subcarrier channel is convex in the subcarrier index $l$ and becomes smaller with the distance between pilot subcarrier and data subcarrier increasing. Since $\sigma_{\hat{h}_{l}}^{2}$ is more close to 1 , the BER performance of the $l^{\text {th }}$ data subcarrier channel is better, as shown in Fig.6. In addition, the optimal energy allocation factor for different data subcarrier channel is also around $a=5$, in Fig.6.

According to Fig.4, 5, 6, 7, we can conclude that when employing generalized linear interpolation technique, the optimum value of $a$, which will result in the minimum BER for SM-OFDM system, is $a^{*}$ which will also results in the minimum BER for a specific subcarrier channel in SM-OFDM system.

Finally, in Fig.7, we compared the simulated BER of SM-OFDM system employing the optimal power allocation with that of employing equal power allocation between the data and pilot symbols. According to the results we

135 observe that when $\mathrm{BER}=10^{-2}$, about $3 \mathrm{~dB}$ gain can be obtained by employing the proposed optimal power power allocation factor for $N_{t}=N_{r}=2$ as well as $N_{t}=N_{r}=4$.

\section{Conclusions}

In this paper, the optimal power allocation between the data and the pilot symbols, which minimizing the upper bound for the BER of SM-OFDM system operating with imperfect CSIR, has been derived. Furthermore, we proved the above optimal allocation is not change with different generalized linear interpolation technique. Finally, the simulation results show that a good gain can 
be obtained for BER performance of SM-OFDM by employing the proposed

optimal power power allocation, comparing to that of employing equal power allocation.

\section{Acknowledgment}

The authors would like to thank the editors and the anonymous referees for their valuable comments and suggestions. This work was supported by the Research Foundation for Advanced Talents of Chengdu University of Information Technology (KYTZ201407), the National Science Foundation of China under Grant number 61471090, the Fundamental Research Funds for the Central Universities (Grant number ZYGX2013J112), the open research fund of National Mobile Commu nications Research Laboratory, Southeast University (No. 2015D09).

\section{References}

[1] R. Mesleh, H. Haas, S. Sinanović, C. W. Ahn, and S. Yun, "Spatial modulation," IEEE Trans. Veh. Technol., vol. 57, no. 4, pp. 2228-2241, July 2008.

[2] M. Di Renzo, H. Haas, and P. M. Grant, "Spatial modulation for multipleantenna wireless systemsA survey," IEEE Commun. Mag., vol. 49, no. 12, pp. 182-191, Dec. 2011.

[3] M. D. Renzo, H. Haas, A. Ghrayeb, S. Sugiura, and L. Hanzo, "Generalized MIMO: Challenges, opportunities, and implementation," Proc. IEEE, vol. 102, no. 1, pp. 56-103, Jan. 2014.

[4] P. Yang, M. Di Renzo, Y. Xiao, S. Li, and L. Hanzo, "Design guidelines for spatial modulation," IEEE Commun. Surveys Tuts., vol. 17, no. 1, pp. 6-26, 1st Quart. 2015. 
[5] S. Ganesan, R. Mesleh, H. Haas, C. W. Ahn, and S. Yun, "On the performance of spatial modulation OFDM," in Proc. 40th Asilomar Conf. Signals, Syst. Comput., Oct. 29-Nov. 1, 2006, pp. 1825-1829.

[6] P. Yang, Y. Xiao, B. Zhou, and S. Li, "Initial performance evaluation of spatial modulation OFDM in LTE-based systems," in Proc. ICST Conf. Harbin, Aug. 2011, pp. 102-107.

[7] T. Kim and J. Andrews, "Optimal pilot-to-data power ratio for MIMOOFMD," Proc., IEEE Globecom, vol. 3, pp. 1481C1485, 2005.

[8] T. Kim, J. G. Andrews, "Balancing Pilot and Data Power for Adaptive MIMO-OFDM Systems," IEEE GLOBECOM 2006, Nov. 2006, pp. 1C5.

[9] R. Mesleh, S. Ganesan, H. Haas, "Impact of Channel Imperfections on Spatial Modulation OFDM," The 18th Annual IEEE International Symposium on Personal, Indoor and Mobile Radio Communications (PIMRC07), pp. 1C5, 3-7 September 2007.

[10] P. Hoeher, S. Kaiser, and P. Robertson, "Two-dimensional pilotsymbolaided channel estimation by Wiener filtering," in Proc. 1997 IEEE Int. Conf. Acoustics, Speech, and Signal Processing, Munich, Germany, Apr. 1997, pp. 1845C1848.

[11] Sun, N., Wu, J., "Optimum designs for high mobility systems with channel estimation errors," Proc. IEEE Int. Conf. Communication ICC 2014, June 2014, pp. 5072C5077

190

[12] T. Handte, A. Muller, and J. Speidel, "BER analysis and optimization of generalized spatial modulation in correlated fading channels," in Proc. IEEE Veh. Technol. Conf., Sep. 2009, pp. 1C5. 